\title{
TURBULENT TRANSPORT OF MAGNETIC FIELDS AND THE SOLAR DYNAMO
}

\author{
L.L.KICHATINOV * \\ Astrophysikalisches Institut Potsdam \\ An der Sternwarte 16, D-O-1590 Potsdam, Germany
}

\begin{abstract}
The effect of anisotropic transport of the mean magnetic field by turbulence in a stratified rotating fluid is briefly discussed to find its qualitative correspondence to the observed field redistribution over a solar cycle. A kinematic model of a dynamo in a spherical shell is developed with the transport effect included to assess its importance in a more consistent way. The anisotropic transport brings the simulated field behavior closer to that observed on the Sun.
\end{abstract}

\section{Anisotropic field transport}

This is a long knowledge (Spitzer 1957; Zeldovich 1957) that apart from the famous $\alpha$-effect and eddy diffusion the turbulence can produce the mean magnetic field transport. The transportation effects contribute the averaged induction equation through the advection-type terms, $\operatorname{rot}\left(\mathbf{V}_{\text {eff }} \times \mathbf{B}\right)$, describing the field propagation with some effective velocity, $\mathbf{V}_{e f f}$, which is not a real velocity of the fluid.

This paper is aimed to draw attention to the turbulent transport effects which are nearly completely disregarded in solar dynamo models. However, they seem to deserve some attention and may be useful in getting agreement between the simulated and observed field dynamics. The motivation is that due to the nearconstancy of solar rotation with depth (cf., e.g., Libbrecht 1989) some mechanism of the mean field transport complementary to the dynamo wave propagation over isorotational surfaces is probably necessary to reproduce the observed latitudinal field migration.

In this respect, a promising feature of the turbulent field transport in rotating fluids is its anisotropy: the effective velocities depend on the field orientation and possess finite horizontal components (Kichatinov 1988, 1991). The anisotropic transport contribution to the mean EMF is

$$
\mathcal{E}_{\text {trans }}=\left(\mathbf{V}^{t} \times \mathbf{B}\right)+\Omega^{-2}\left(\left(\mathbf{V}^{p}-\mathbf{V}^{t}\right) \times \mathbf{\Omega}\right)(\boldsymbol{\Omega} \cdot \mathbf{B})
$$

where $\Omega$ is the angular velocity. For the axi-symmetric fields, to which case we shall limit our consideration, the $\mathbf{V}^{p}$ and $\mathbf{V}^{t}$ in (1) are the effective velocities for the poloidal and toroidal field components.

The transportation effects exist for inhomogeneous fluids only. Between the two basic inhomogeneities of density and turbulence intensity the latter is probably more important for the Sun. We consider the transport velocities for the rapid rotation case $\left(\Omega^{*}=2 \tau \Omega \gg 1\right.$ with $\tau$ being the convective turn-over time),

$$
\mathbf{V}^{t}=\eta_{t}\left(\mathbf{G}-2 \mathbf{G}_{\perp}\right) / 2, \quad \mathbf{V}^{p}=\eta_{t}\left(\mathbf{G}+\mathbf{G}_{\perp}\right) / 2
$$
Russia

* On leave from the Institute for Solar-Terrestrial Physics, P.O. Box 4026, Irkutsk 664033, 
where $\mathbf{G}=\nabla \ln (\rho)$ is the relative density gradient, $\mathbf{G}_{\perp}$ means its normal component to the rotation axis,

$$
\eta_{t}=\tau \pi<u^{2}>/\left(8 \Omega^{*}\right)
$$

is the isotropic part of the effective magnetic diffusivity for the rapid rotation case (Kichatinov 1988). The case $\Omega^{*} \gg 1$ is chosen partly by the reason of simplicity and partly to show that the decrease of the transportation velocities due to rotational quenching does not cancel the effect because the eddy diffusivity is equally quenched. The Sun with $\Omega^{*} \simeq 6$ (Durney and Latour 1978) is nearly a rapid rotator.

The velocities (2) describe the poloidal field transport to poles which poleward migration is really observed, at high latitudes at least. The toroidal field is transported to equator in qualitative agreement with the sunspot activity migration. Next, the radial component of the toroidal field velocity is negative (downward) at high latitudes and positive (upward) at low latitudes. This may be an explanation of why the sunspots are seldom at high latitudes: the toroidal fields may be locked there deep in the convection zone due to the downward transport. The estimations of the transportation times also give reasonable solar values (Kichatinov 1991). Thus, with the anisotropic transport effect different lines of observational evidence can be interpreted from a common point of view. This consideration seems promising but somewhat superficial however. Indeed, the toroidal and poloidal fields are physically related to each other (through the $\alpha$-effect and differential rotation). It is not obvious that the physical ties can allow the anisotropic transport to redistribute the fields 'as it wants'.

\section{Dynamo model}

A more consistent way to assess the importance of the anisotropic turbulent transport is probably to include it in a dynamo model. Than a comparison of the results of the runs made with/without inclusion of this effect can show whether it is significant.

A relatively simple linear and axisymmetric model of the $\alpha \Omega$-dynamo in a spherical shell was used to this end. The normalized equations,

$$
\begin{aligned}
\frac{\partial b}{\partial t}= & -P \frac{2}{x}\left(\frac{\partial f}{\partial x} \frac{\partial a}{\partial \theta}-\frac{\partial f}{\partial \theta} \frac{\partial a}{\partial x}\right)+\frac{1}{x^{2}} \frac{\partial}{\partial \theta} \frac{1}{\sin \theta} \frac{\partial}{\partial \theta} \sin \theta b+\frac{1}{x} \frac{\partial^{2} x b}{\partial x^{2}} \\
& +\frac{\xi}{2 x}\left(\left(1-2 \sin ^{2} \theta\right) \frac{\partial x b}{\partial x}-2 \frac{\partial}{\partial \theta} \sin \theta \cos \theta b\right) \\
\frac{\partial a}{\partial t}= & x \sin \theta \cos \theta b+\frac{\sin \theta}{x^{2}} \frac{\partial}{\partial \theta} \frac{1}{\sin \theta} \frac{\partial a}{\partial \theta}+\frac{\partial^{2} a}{\partial x^{2}} \\
& +\frac{\xi}{2}\left(\left(1+\sin ^{2} \theta\right) \frac{\partial a}{\partial x}+\cos \theta \sin \theta \frac{1}{x} \frac{\partial a}{\partial \theta}\right)
\end{aligned}
$$

include the anisotropic transport effect by their second lines. In this equations, $x=$ $r / R$ is the fractional radius, $b$ and $a / x \sin \theta$ are toroidal field and the poloidal field potential respectively, $\xi=R G$ is a stratification parameter, $P=-\alpha_{0} \Omega_{0} R^{3} /\left(2 \eta_{t}^{2}\right)$ 
is the dynamo number, and $f(x, \theta)$ is the normalized angular velocity:

$$
\Omega(x, \theta)=\Omega_{0} f(x, \theta) .
$$

Several simplifying assumptions are adopted in (4). The eddy diffusivity is also anisotropic under rapid rotation; this anisotropy is ignored and the diffusivity coefficient (3) is assumed constant. The coefficient $\alpha$ of the $\alpha$-effect is taken constant with depth and latitude dependent as $\cos \theta$. The stratification parameter is also assumed constant, the value $\xi=20$ was used in the computations. The angular velocity distribution adopted is depth-independent rotation from the top to the middle of convection zone and the disc-shaped rotation isoplanes from the middle to the bottom:

$$
f(x, \theta)=\left\{\begin{array}{cl}
1-\left(x / 2 x_{1}\right)^{2} \cos ^{2} \theta & \text { for } x_{0} \leq x \leq x_{1} \\
1-\cos ^{2} \theta / 4 & \text { for } x_{1} \leq x \leq 1,
\end{array}\right.
$$

where $x_{0}$ and $x_{1}=x_{0}+\left(1-x_{0}\right) / 2$ are the radii of the bottom boundary and of the middle of the shell respectively. The value $x_{0}=0.5$ was used in the computations.

As usual, the linear dynamo model was reduced to the eigenvalue problem and the latitude dependences were represented as the series expansions in the adjoint Legendre polynomials:

$$
a=\exp (\lambda t) \sum_{n} a_{n}(x) \sin \theta P_{n}^{1}(\cos \theta), \quad b=\exp (\lambda t) \sum_{n} b_{n}(x) P_{n}^{1}(\cos \theta) .
$$

14 harmonics were kept in the expansions (6). A uniform grid over the variable $x$ with 21 grid points was used.

The usual boundary conditions were imposed corresponding to vacuum outside the spherical shell,

$$
b_{n}=0, \quad \partial a_{n} / \partial x=-n a_{n} \text { for } x=1,
$$

and a superconductor below it,

$$
a_{n}=0, \quad x \xi\left(1-2 \sin ^{2} \theta\right) b+2 \partial(x b) / \partial x=0 \quad \text { for } x=x_{0} .
$$

\section{Some results and discussion}

Only negative dynamo numbers are considered because this sign is expected for the Sun. The linear stability diagram is shown in Fig. 1. The modes with dipol and quadrupol parities have nearly the same critical dynamo numbers, $P_{c r}$, defined by the equation, $\operatorname{Re}\left[\lambda\left(P_{c r}\right)\right]=0$. Still, the $P_{c r}$ for dipol parity is a bit smaller (in absolute value). We consider only dipol parity modes because they dominate on the Sun (Stenflo 1988).

Fig. 2 shows the simulated butterfly diagram and isocontours of the radial component of the field on the upper surface for the critical dynamo number. The same diagrams found with the anisotropic transport neglected are shown in Fig. 3. On comparing these two figures, we can notice that the anisotropic turbulent transport does not produce any dramatic changes. Nevertheless, the changes are quite pronounced and they are generally to improve agreement with observations. Indeed, 


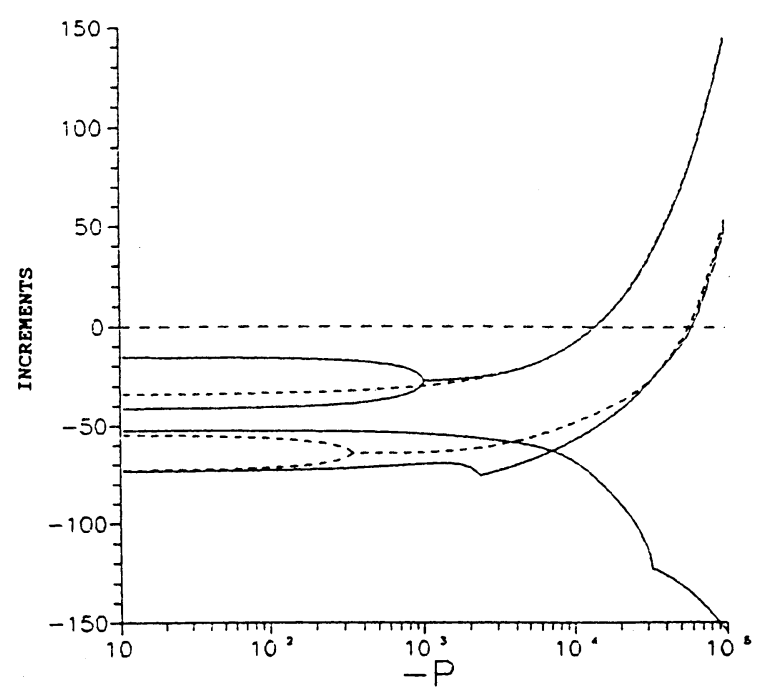

Fig. 1. The dependences of the real parts of the eigenvalues on the dynamo number. The full and broken lines show the results for dipol and quadrupol parities respectively

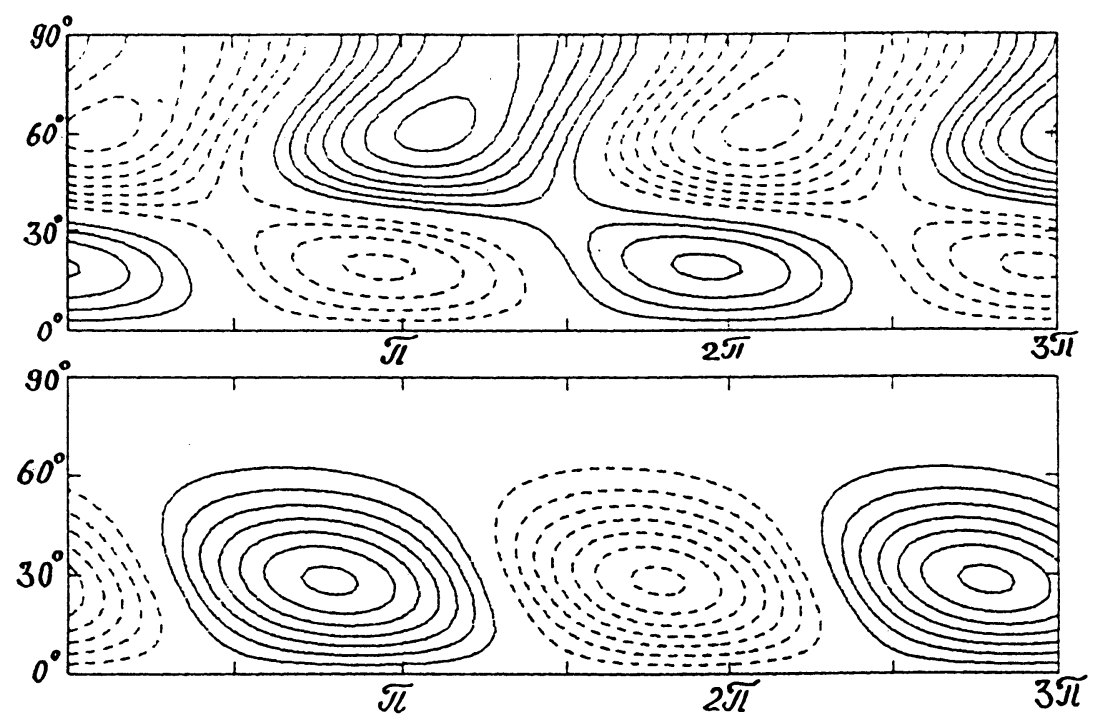

Fig. 2. Butterfly diagram for toroidal field at a small depth below the surface $(x=0.9)$ in coordinates latitude-phase of the dynamo cycle (lower panel) and the contour-lines for the radial component of the field on the surface (upper panel). The full and broken lines show positive and negative levels respectively. For this case, $P_{c r}=-1.40 \cdot 10^{4}$ and the critical frequency $\omega_{c r}=\operatorname{Im}\left[\lambda\left(P_{c r}\right)\right]=65.4$ 

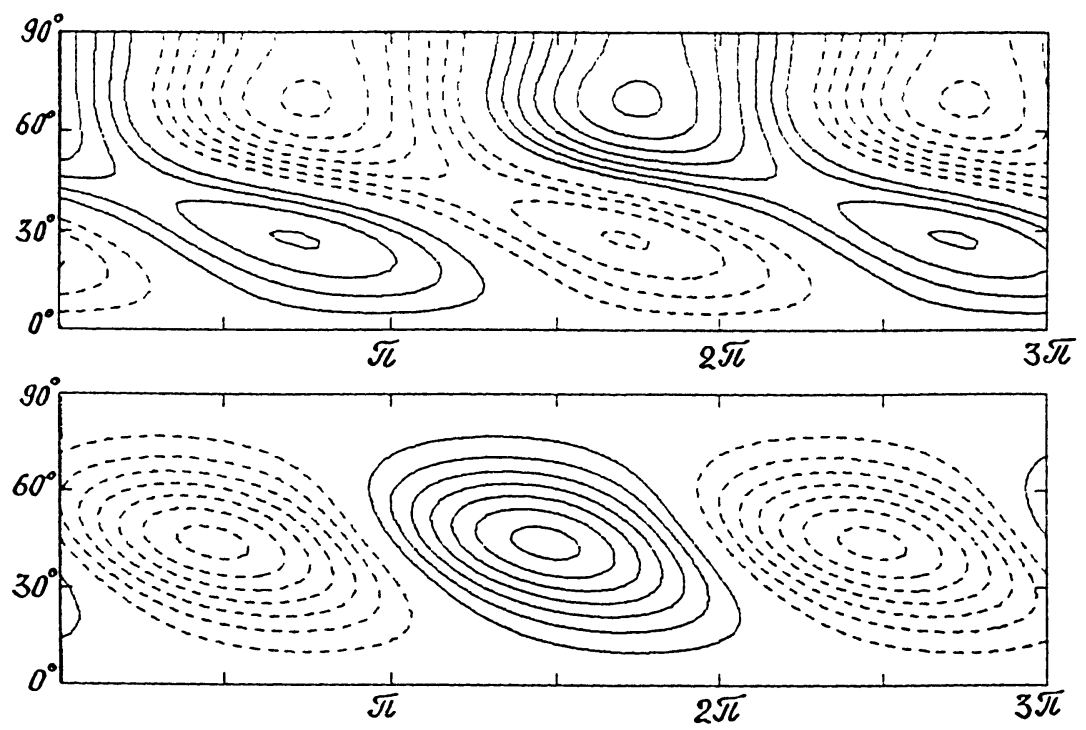

Fig. 3. The same as in Fig. 2 but with the turbulent transport neglected. $P_{c r}=-1.18 \cdot 10^{4}$ and $\omega_{c r}=79.2$ for this case

the butterfly diagram of Fig. 2 has narrower 'wings', though still somewhat too wide, and is closer to the observational picture. Next, the poloidal field isocontours of Fig. 2 are rather similar to the diagram found by Stenflo (1988). Note that the turbulent transport produces a polar branch of the poloidal field which branch is quite pronounced in the observational diagram by Stenflo (1988). No such a branch exists in Fig. 3 found with the anisotropic transport neglected.

We can notice, however, that a very small radial inhomogeneity of rotation law (5) produces quite noticeable latitudinal field migration in Fig. 3. The anisotropic turbulent transport is certainly not the only process governing the latitudinal field redistribution over a dynamo-cycle but it is important.

There are probably two main points to conclude:

1. The turbulent transport of the mean magnetic fields in a density-stratified rotating fluid is anisotropic. In particular, the transport velocities for poloidal and toroidal components of an axisymmetric field are different and possess finite horizontal components.

2. The anisotropic transport influences the dynamo modes generally to improve agreement with observations.

\section{References}

Durney, B.R. and Latour, J: 1978, Geophys. Astrophas. Fluid Dyn. 9, 241

Kichatinov, L.L.: 1988, Astron. Nachr. 309, 197

Kichatinov, L.L.: 1991, Astron. Astrophys. 243, 483

Libbrecht, K.G.: 1989, Astrophys. J. 336, 1092

Spitzer, L.: 1957, Astrophys. J. 125, 525

Stenflo, J.O.: 1988, Astrophys. Space Sci. 144, 321

Zeldovich, Ya.B.: 1957, Sov. Phys. JETP 4, 460 